\section{MR-PET opens new horizons in neuroimaging}

\author{
Hans Herzog ${ }^{\dagger 1}$, Karl-Josef Langen', Joachim Kaffanke', Christoph Weirich', \\ Irene Neuner',2, Gabriele Stoffels', Elena Rota Kops', Jürgen Scheins', \\ Lutz Tellmann' \& Nadim Jon Shah',3

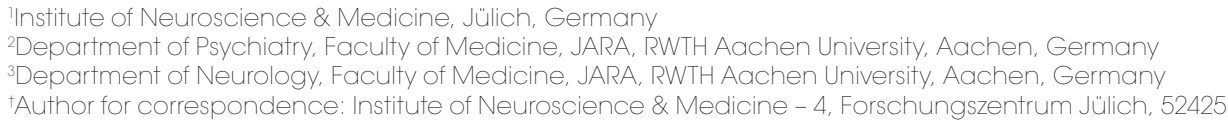

At present, a new hybrid imaging technology combining PET and MRI is emerging. The construction of hybrid scanners that allow simultaneous MRI and PET required the development of a new type of MR-compatible PET detector, which was first realized for studying small animals. The first industrial prototypes of such integrated MR-PET scanners for human brain studies have been installed recently. In this article, the feasibility of this new approach is demonstrated by first studies in brain tumor patients. Beyond time saving in patient management and optimal spatial overlay of anatomic and functional information, this technique offers new perspectives with regard to the simultaneous observation of physiological and biochemical processes. Functional brain activation and interaction of receptor ligands during special activation tasks or pharmacological intervention may be explored in humans. Thus, MR-PET opens new horizons in multiparametric neuroimaging.

The different tomographic methods available in medical diagnostics and research deliver comprehensive structural and functional insights of the body. Whereas CT and MRI primarily produce high-resolution images of anatomical details, single photon emission CT (SPECT) and PET allow imaging of a wide variety of physiological and biochemical processes. Previously, patients had to be investigated sequentially by these methods to gather complementary diagnostic information. The introduction of PET and CT in a single device provides corresponding functional and structural information in one examination [1]. Especially in oncology, the combination of CT with PET using $\left[{ }^{18} \mathrm{~F}\right]$-fluorodeoxyglucose has proved to be most successful [2-4]. As a consequence, industry shows increasing interest in combining other tomographic devices in order to disclose new perspectives for research as well as clinical applications. SPECT/CT, originally suggested by Hasegawa [5] and nowadays offered by the majority of manufacturers, is becoming more and more popular $[2,3,6]$. Recently, the first prototypes of hybrid scanners allowing simultaneous MR-PET for brain studies have been developed and installed in two centers in both Germany and the USA. The combination of the outstanding diagnostic properties of MRI and PET enables a variety of totally novel multiparametric applications if both methods can be performed simultaneously. Whereas PET/CT and SPECT/CT could be combined using existing technologies, the integration of PET within an MRI device demands a new detector technology for PET. In this article the development of this new technology is explained, the technical features of MR-PET are described, the results of first applications in humans are presented and possible applications are indicated.

\section{Towards PET integrated with ultra-high field MRI for small animal studies}

The first experimental work that resulted in simultaneously acquired PET und MR images of a phantom was pioneered by the group of Cherry and Marsden at University of California, Los Angeles (UCLA; CA, USA) in 1997 [7]. To avoid interferences from the magnetic field of a $0.2 \mathrm{~T}$ open magnet MRI system onto the magnetosensitive PET electronics, they connected the scintillation crystals and the photomultiplier by 4-m long optical fibers. If the PET detector of a MR-PET system utilizes the classical detector technology with photomultipliers, these electronic tubes must be kept away from the magnetic field. Furthermore, if only the scintillation crystals are in the MR field of view, interferences of the PET detector onto MRI are minimized. Consequently, more recent approaches kept the photomultiplier outside the magnetic field and coupled them to scintillation crystals by optical fibers of least $0.8 \mathrm{~m}$ [8-11]. A direct coupling without fibers became possible in the case of a field-cycled MR scanner [12]. Until now most of
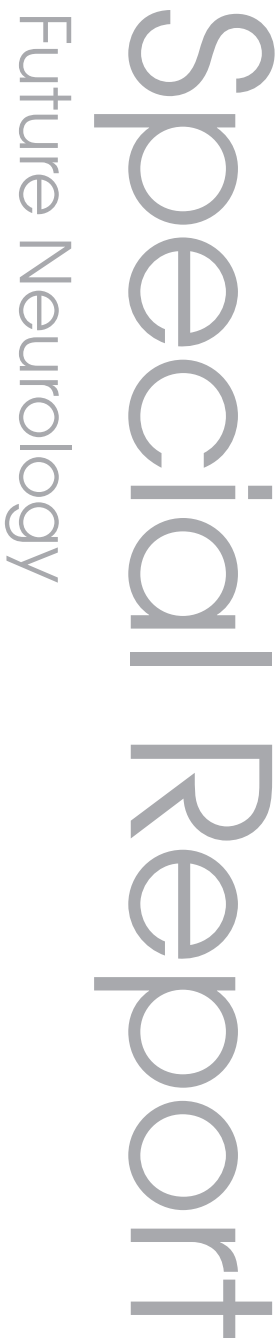

\section{Keywords}

- hybrid imaging $=$ magnetic resonance imaging $=$ MRI - MR-PET $=$ multimodality - $\mathrm{PET}$ - $\mathrm{PET} / \mathrm{MRI}$ - positron emission tomography

\section{fulure medicine $^{\text {pis }}$ ss}


these developments designed for small animal imaging are still ongoing and being tested for further optimization.

Since light loss along the fibers compromises the quality of PET imaging, solutions had to be found that allow very short fibers or a direct coupling between scintillation crystals and readout electronics located very near or in the magnetic field of conventional MR scanners. The fundamental solution was the replacement of photomultipliers by solid state components, such as Avalanche photodiodes (APDs) as first suggested by Pichler and coworkers in 1998 [13], and later realized by Pichler, Catana and coworkers in Cherry's group at the University of California, Davis (CA, USA) [14,15]. Catana reported a PET detector where fibers of approximately $15 \mathrm{~cm}$ in length coupled an $8 \times 8$ array of lutetium oxyorthosilicate (LSO) crystals to a position-sensitive APD [15]. This detector was placed in a Bruker 7-T Biospec animal MRI system. PET images of a mouse after injection of $\left[{ }^{18} \mathrm{~F}\right]$-fluoride could be recorded ex vivo simultaneously with MR images without showing remarkable artefacts. In parallel, this approach has been and is being further developed by Pichler's group at the University of Tübingen (Tübingen, Germany), which achieved a direct coupling between LSO crystals and an array of single APDs within a small animal 7-T MR scanner [16]. At different meetings and in initial publications this group reported successful hybrid MR-PET imaging in mice and rats $[17,18]$.

Schlyer and coworkers at Brookhaven National Laboratories (NY, USA) [19] constructed a small APD-based detector ring known as 'Rat Conscious Animal PET' (RatCAP), which can be fixed around the neck and head of a rat in a way that the animal carries the PET ring and can remain conscious. Using APDs to read out the LSO crystals the first RatCAP was already basically MR compatible. After different modifications the PET ring can be operated within a 9.4 T MRI for small animals, which must, however, been anesthetized. Recently, first studies after injection of the dopamine radioligand $\left[{ }^{11} \mathrm{C}\right]$-raclopride in a rat could demonstrate the feasibility of the RatCAP-based approach [20].

First prototypes of a MR-PET scanner for human brain studies

In November 2006 the first PET and MR images simultaneously acquired in a human subject were presented at the meeting of the Radiological Society of North America (RSNA) [21]. These images were obtained with a new APD-based PET detector known as BrainPET and developed by Siemens (Munich, Germany) [22]. The BrainPET is operated within a standard Siemens 3T MAGNETOM Trio MR scanner (Figure 1). The basic design of the PET detector is similar to that of Pichler's group. The front end of the detector module is a $12 \times 12$ matrix of $2.5 \times 2.5 \times 20 \mathrm{~mm}^{3}$ individual LSO crystals coupled to a $3 \times 3$ array of APDs. Six detector modules are aligned in a copper-shielded cassette, 32 of which form the cylindrical PET detector, which has an outer diameter of $60 \mathrm{~cm}$ just fitting the MR bore. Two dedicated head coils for MR (one outer bird cage coil for combined transmit and receive, and one inner eight-channel coil for receive), which are both optimized in regard to minimal attenuation for PET, can be placed into the PET ring with an inner diameter of $32 \mathrm{~cm}$. The transmit/receive coil is necessary because the normal whole-body transmit coil of the MAGNETOM Trio is switched off in the 3TMR-BrainPET. Each of the 32 cassettes is linked by a $10-\mathrm{m}$ long cable to the filter plate of the MRI cabin, on the exterior of which a modified version of the QuickSilver data acquisition electronics [23] is attached. The coincidence window of the BrainPET is $12 \mathrm{~ns}$ and the energy window ranges from 420 to $580 \mathrm{keV}$. Since the APDs are very temperature dependent, the BrainPET's temperature is stabilized with cooled air. Preliminary performance tests confirmed the feasibility for simultaneous MR-PET imaging without severe interferences between the two modalities and some favorable performance parameters that are equal to or better than those of other present PET detectors for human studies [24]. The central image resolution is approximately $3 \mathrm{~mm}$, which means the BrainPET together with the high-resolution research tomograph PET-only scanner [25] offers the best PET image resolution presently available for human brain studies. The count rate does not saturate at radioactivities applied for human studies and the (point source) sensitivity is approximately $6 \%$. Since the BrainPET is an insert of the MR scanner and not integrated within the MR gantry, it might even be operated alone. From 2006 to 2010 a limited number of four prototypes of this 3TMR-BrainPET have been installed in two German laboratories at Tübingen and Jülich and in Boston and Atlanta, where they have been undergoing comprehensive tests. 


\section{First human studies with the 3TMR-BrainPET in Jülich}

The results obtained with the 3TMR-BrainPET to date, and reported at scientific meetings and in first publications, aim at examining the feasibility of the integrated MR-PET scanner by comparing images of BrainPET with that of conventional PET or PET/CT scanners. The primary aim of testing the 3TMR-BrainPET is to look at the performance of the newly designed PET detector operated in the specific environment of a magnetic field. The first tests of the 3TMR-BrainPET demonstrated that different MR sequences can be conducted simultaneously with the PET measurement without revealing obvious MR artefacts. Nevertheless, after the ongoing tests of the BrainPET the MR performance will be tested in more detail.

In our laboratory the evaluation of the 3TMRBrainPET is primarily based on comparative studies of patients with malignant brain tumors who are primarily scanned in an ECAT Exact HR+PET scanner for diagnostic purposes or for a clinical research study, for example to look at therapeutic effects [26]. Since the 3TMR-BrainPET is not a commercial product but a prototype, it can only be applied for human studies under the conditions of a clinical study according to $\$ 20$ of the German Medical Devices Act. The studies were approved by the local ethical committee and written informed consent was obtained from each patient measured in the 3TMR-BrainPET.

The study with the HR+- PET was recorded for $50 \mathrm{~min}$ after the injection of approximately $200 \mathrm{MBq}\left[{ }^{18} \mathrm{~F}\right]$-fluoroethyltyrosine (FET), an amino-acid tracer that is able to delineate the tumor better than MRI [27]. After this scan the patient may have been asked to volunteer for a further imaging procedure of $30-40 \mathrm{~min}$ in the 3TMR-BrainPET. For this scan no additional FET needed to be injected, because the biological washout of FET out of the brain and tumor as well as the radioactive decay with a physical halflife of 109 min are slow. Thus, the BrainPET images were compared with the results of the HR+ as demonstrated in Figure 2. The images of the two scanners are very similar. Small mismatches of the spatial distribution of FET can be explained by the fact that the radiotracer's uptake is not stationary in normal brain and tumor, but changes in a different way. In order to achieve comparable count statistics, the summed HR+ image comprises $20 \mathrm{~min}$, whereas the BrainPET image acquired later was summed over $30 \mathrm{~min}$. Both PET images show the tumor clearly. The BrainPET images look less blurred than the HR+

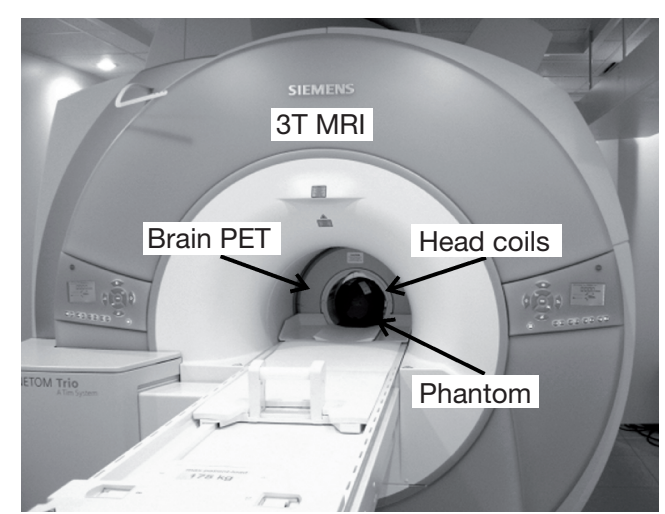

Figure 1. The 3TMR-BrainPET as installed in the Forschungszentrum Jülich, Germany.

The BrainPET is constructed as an insert into the MR bore. The MR head coils just fit into the BrainPET.

images, indicating the better image resolution of the BrainPET. During the BrainPET scan shown in this article a number of MR sequences were recorded: $T_{1}$-weighted magnetization-prepared rapid acquisition with gradient echo (MPRAGE) with and without contrast enhancement using gadolinium and $\mathrm{T}_{2}$-weighted turbo inversion recovery magnitude (FIGURE 3). These recordings, together with the initial localizer, took approximately 20 min so that there is ample time for additional sequences during the PET acquisition, as indicated later.

Since the PET images are not only analyzed qualitatively, but also quantitatively, for example by determining the tumor-to-brain ratio of FET uptake at a certain time after injection, the PET data must undergo several corrections. In conventional PET and PET/CT, transmission scans are performed to measure a tissue attenuation map needed for attenuation correction. This is not possible in the 3TMR-BrainPET, because the spatial conditions do not allow the installation of a radiation source needed for transmission scans. Therefore, different solutions have been suggested and implemented to derive attenuation maps from the anatomic information offered by MRI [28-32]. In our group we have developed a template-based method [29] using a template of an MPRAGE image averaged from eight scans of different subjects, which were spatially normalized [33] to one subject's head shape and a corresponding template of an attenuation map obtained from transmission scans of these subjects recorded with the HR+- PET (Figure 4). In addition, the MR coils must be included into the attenuation map [29,32]. Beside adequate attenuation correction, further corrections for detector efficiency (normalization), radioactive decay, dead time and scatter, as well as calibration of 


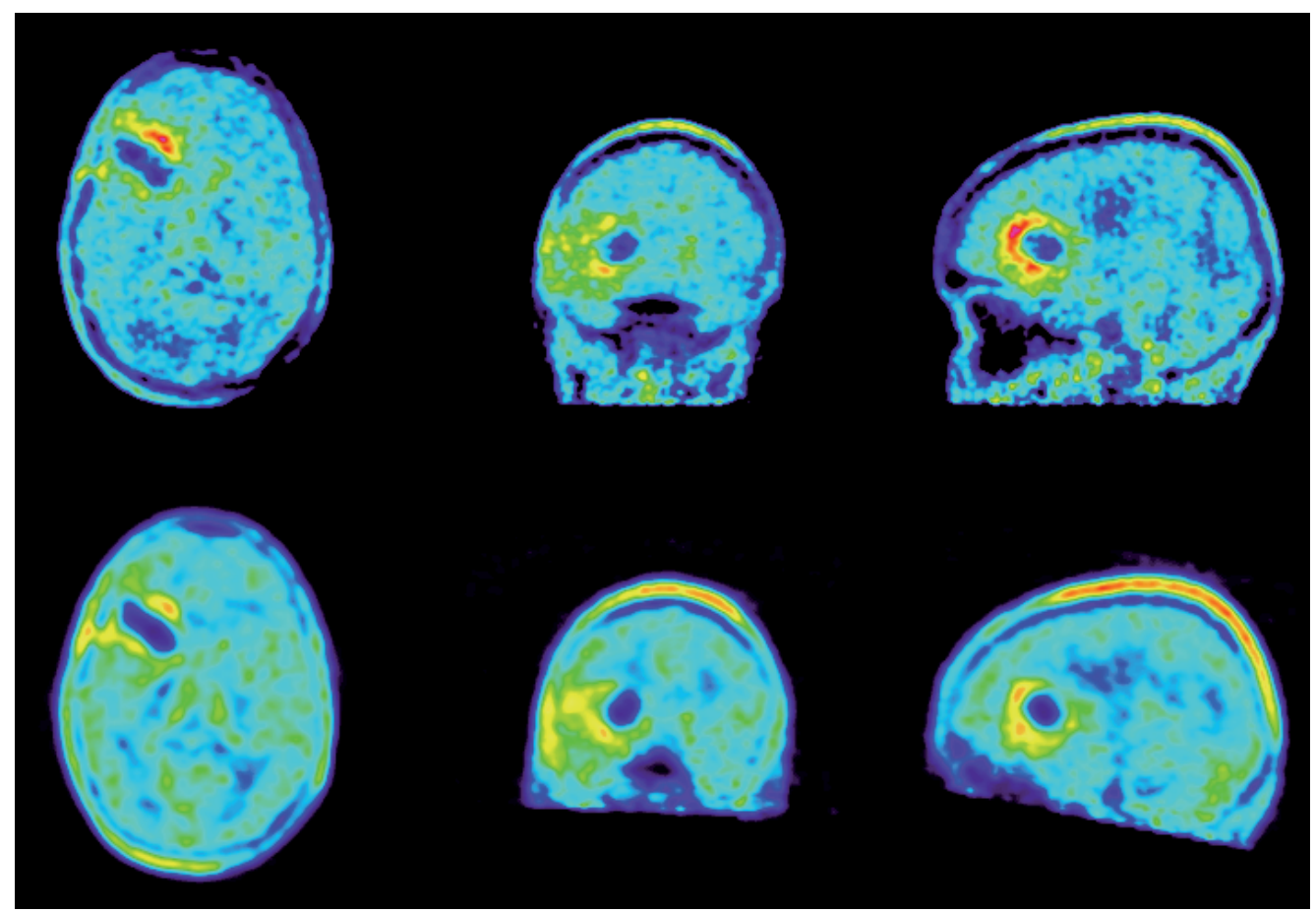

Figure 2. Transversal, coronal and sagittal images (from left to right) of a patient with a glioblastoma after surgery. The images on the top row were recorded with the BrainPET from 94 to $124 \mathrm{~min}$ after injection of approxiamtely $200 \mathrm{MBq}\left[{ }^{18} \mathrm{~F}\right]$-fluoroethyltyrosine. The images on the bottom row were recorded with the HR+- PET and summed from 20 to 40 min after radiotracer injection.

the BrainPET, are essential to produce quantitative images. The capability of the BrainPET to produce quantitative scans could be demonstrated in our laboratory in a number of patient studies by comparing BrainPET-based ratios of regions of interest of tumor and normal cortex and corresponding time-activity curves with those of the preceding HR+- scan. Thereafter, the protocol was changed in some patients who were placed first in the BrainPET so that dynamic measurements in the MR-PET could be performed after tracer injection. An example of such a patient who was referred with a suspicion of a low-grade tumor is demonstrated in Figures 5 \& 6 . FET was injected intravenously into the patient lying in the 3TMR-BrainPET and a PET scan of $50 \mathrm{~min}$ was started, which delivered images on the FET kinetics similar to those observed with the HR+scanner. After a short interval a complementary scan for 30 min was performed with the HR+PET. During the BrainPET acquisition, simultaneous MRI was performed. In addition to the standard MR sequences, chemical shift imaging (CSI) was performed focused to the lesion visualized by the MPRAGE image (Figure 5B), which was recorded directly after tracer injection. Figures $5 \mathrm{~A} \&$ 6 display the BrainPET image summed from 20 to $40 \mathrm{~min}$ after injection and the time-activity

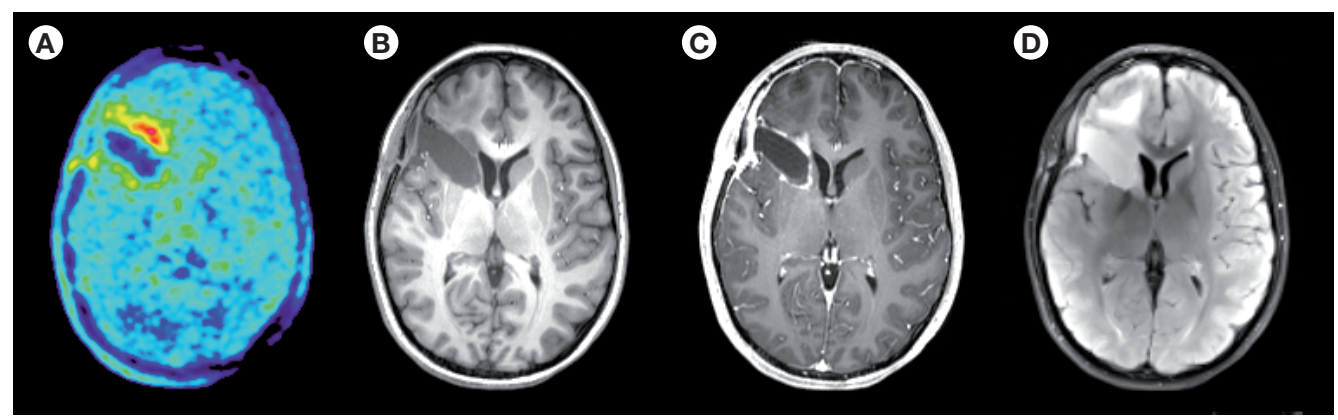

Figure 3. PET simultaneous with versatile MRI. (A) While the patient already shown in FIGURE 2 was investigated by $\left[{ }^{18} \mathrm{~F}\right]-f l u o r o e t h y l t y r o s i n e-B r a i n P E T$, three MR sequences were performed:

(B) $T_{1}$-weighted magnetization-prepared rapid acquisition with gradient echo (MPRAGE) without contrast medium; (C) T -weighted MPRAGE with contrast medium; and (D) T2-weighted turbo inversion recovery magnitude. 


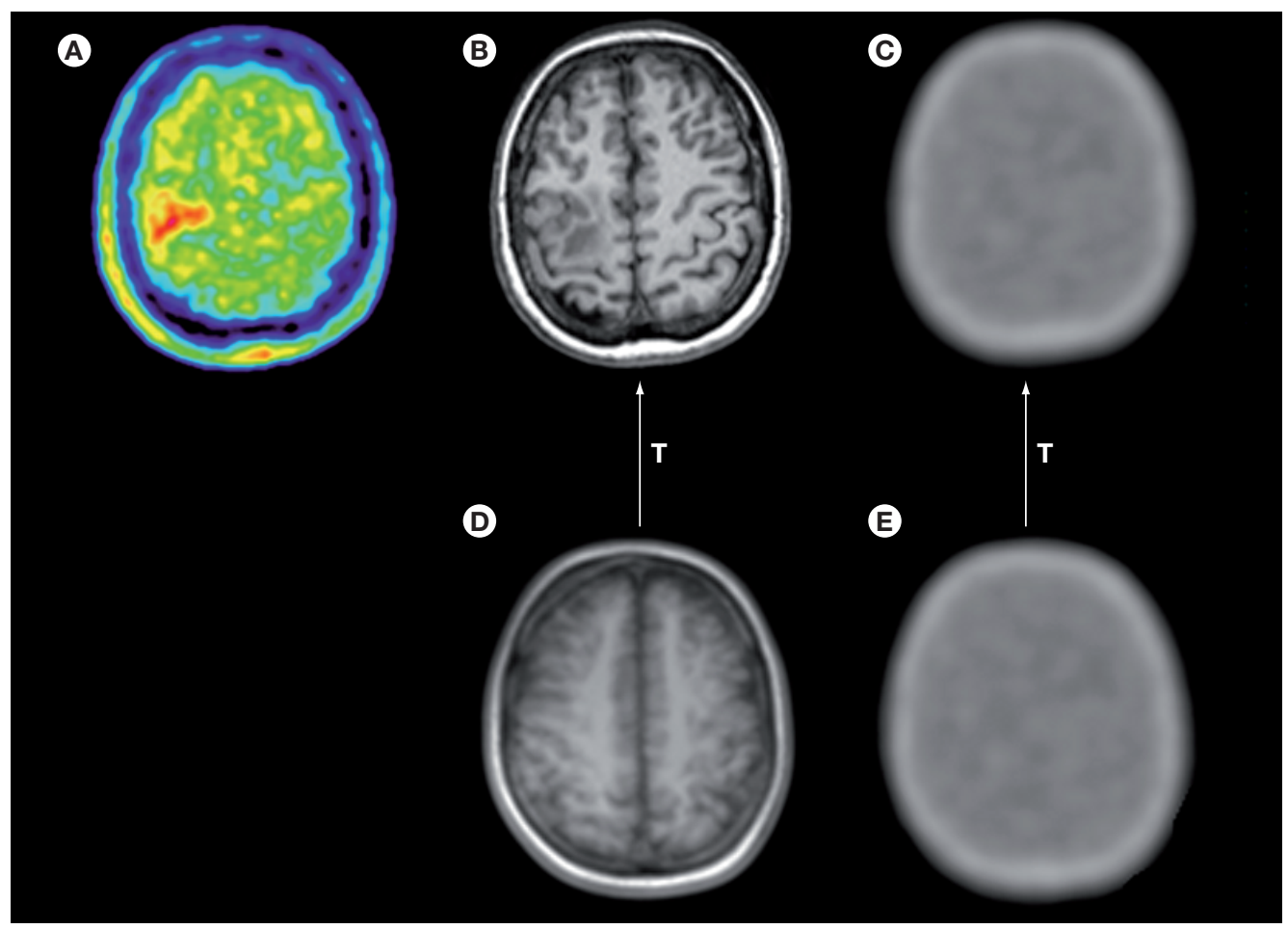

Figure 4. Different steps of the template-based method to obtain a patient-specific attenuation map. (A) The 3TMR-BrainPET measurement delivers the PET data and (B) the magnetization-prepared rapid acquisition with gradient echo (MPRAGE) image of a patient. Using the Statistical Parametric Mapping (SPM) software [33] the matrix T describing the deformation from the MPRAGE template (D) to (B) is determined. Then T is applied to the attenuation template (E) resulting in the attenuation map $(\mathbf{C})$ specific for the individual patient.

curves of regions of interest placed over the lesion and the normal cortex. Both the image and the time-activity curves show no differences between lesion and normal cortex so that the diagnosis of a high-grade tumor was unlikely. Conversely, CSI revealed an abnormal spectrum at the site of the lesion, which differed from that of the contralateral normal tissue. A parametric map (Figure 5C) resulting from CSI presented an increased ratio of myo-inositol and $N$-acetyl-aspartate, which may also indicate an inflammatory process [34]. Thus, the simultaneous MR-PET study was able to deliver bimodal information about the lesion in a single imaging session of just $50 \mathrm{~min}$.

The visual comparison of brain scans obtained with the BrainPET and the $\mathrm{HR}_{+-}$scanner demonstrates the superior image quality of the BrainPET and confirms the experimental data on image resolution given above. This finding is also underlined by a recent paper by Boss and coworkers [35]. They compared brain tumor images recorded with a PET/CT first and thereafter with the BrainPET. Since the resolution of the images of the BrainPET superseded those of the PET/ CT, the authors smoothed the BrainPET's images so that the partial volume effect became the same for both scanners and the final results matched.
In spite of these favorable results a critical inspection of both PET and MR images reveals some minor residual artifacts that need to be removed. The ongoing tests of the 3TMRBrainPET prototypes will try to find out for which applications these subtle problems are relevant or not and how they can be solved.

\section{The near future of MR-PET}

While the prototypes of the 3TMR-BrainPET are being tested, Siemens is developing a wholebody 3TMR-PET hybrid scanner allowing for simultaneous bimodal imaging. Similar to the BrainPET, the PET detector is integrated within the MR scanner, however, not as a separate insert but outside the bore between the gradient and radiofrequency coils. The tests of first prototypes are expected in late 2010. If the performance of this novel scanner type is successful a commercial version can be expected, while at present there is no commitment of the manufacturer to produce a commercial version of the 3TMR-BrainPET. This is owing to the fact that the market for specific brain scanners is regarded as limited. Whereas Siemens' MR-PET systems are integrated scanners, Philips decided to combine its standard 3TMR scanner Achieva with 


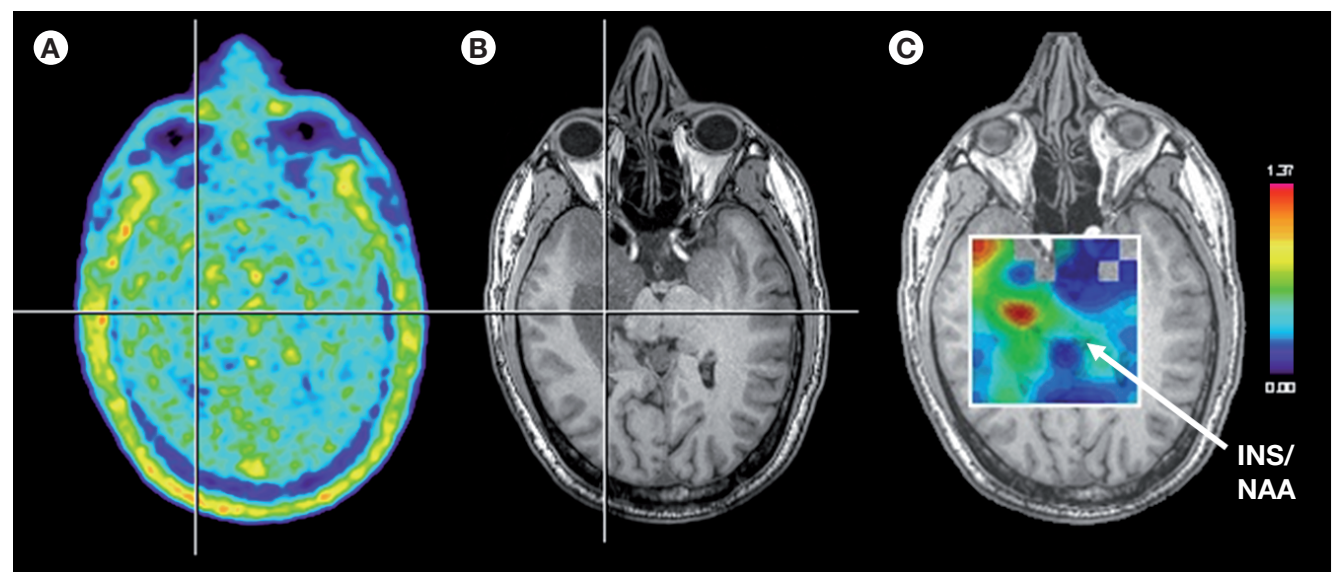

Figure 5. $\left[{ }^{18} \mathrm{~F}\right]$-fluoroethyltyrosine-PET and MPRAGE image of a patient referred with a suspicion of a low grade tumor. (A) $\left[{ }^{18} \mathrm{~F}\right]$-fluoroethyltyrosine-PET revealed no radiotracer uptake at the lesion (cross) visible in (B) the magnetization-prepared rapid acquisition with gradient echo (MPRAGE) image. (C) After the MPRAGE image, chemical shift imaging was recorded while the PET measurement was still ongoing. Chemical shift imaging showed an increased ratio of myo-inositol and $\mathrm{N}$-acetyl-aspartate, indicating the possible involvement of an inflammatory process. INS: Myo-inositol; NAA: N-acetyl-aspartate.

the PET component of their PET/CT. In this hybrid scanner both components are remote from each other by approximately $3 \mathrm{~m}$, but linked by a common patient bed. The system does not allow for simultaneous imaging, but helps to minimize or even avoid misalignments between MR and PET, because the patient does not need to change their beds, keeping his position from one scan to the other. By combining standard MR and PET components and keeping the PET away from the

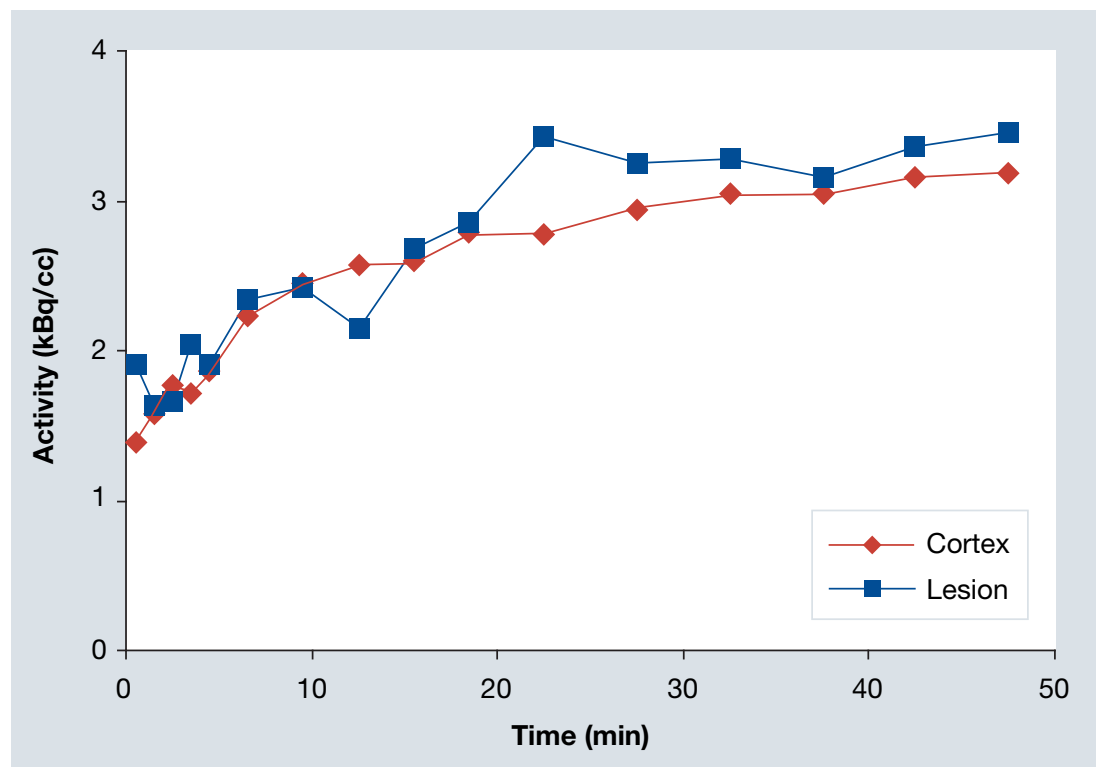

Figure 6. Quantitative $\left[{ }^{18} \mathrm{~F}\right]-$ fluoroethyltyrosine kinetics recorded with the 3TMR-BrainPET. Time-activity curves of [ $\left.{ }^{18} \mathrm{~F}\right]$-fluoroethyltyrosine kinetics in the patient shown in Figure 5. There is practically no difference between the curves of normal brain and the lesion, indicating that the lesion visible in the magnetizationprepared rapid acquisition with gradient echo (MPRAGE) image (FIGURE 5B) is unlikely to be caused by a high-grade tumor. magnetic field (a residual magnetic field is taken into account by appropriate shielding) Philips avoided the intrinsic problems of an integrated MR-PET. Although this hybrid scanner will not allow simultaneous MR-PET imaging, it may deliver valuable data on the performance of wholebody MR-PET in comparison with PET/CT in cancer diagnostics [36]. Furthermore, Philips is exploring the field of simultaneous MR-PET under the EU FP7 project HYPERImage [101]. The goal of this project is the joint development, with various academic and industrial partners, of a new compact digital MR-compatible TOF-PET detector technology, which will be first tested in a prototype of a preclinical scanner for rabbits and mice. There is no commitment by Philips regarding a commercial product, neither for small animal nor human studies.

The necessity of MR-PET allowing simultaneous acquisition of both modalities remains a matter of discussion [37-41]. For the brain, anatomical coregistration is quite reliable and functional PET images can conveniently be registered to anatomical MR images using one of the many fusion programs available from academics and industry. From this point of view one may not need a simultaneous MR-PET recording. On the other hand, in our experience multiparameter imaging with different devices is extremely time consuming and stressful to the patients, and the reduction of scanner times is an important point in patient care. This applies especially for cancer patients and patients with severe neurological disorders. Furthermore, the economic advantage of a more efficient patient throughput needs to be considered. 
In brain research a combined MR-PET offers many perspectives beyond the common combination of functional PET and anatomical MRI. Considering the capabilities of physiological and biochemical MRI, a variety of research investigations can be foreseen in which the functional capabilities of both MRI and PET may be used in a complementary way [42]. For example, the reaction of the brain to pharmacological challenges or mental stimulation may be observed by neuroreceptor studies in parallel with functional activation by functional MRI. Another example, which may even be of clinical relevance for cerebrovascular diseases, is the combined measurement of brain perfusion by diffusionweighted imaging and of oxygen consumption by $\left[{ }^{15} \mathrm{O}\right]$-oxygen-PET.

Thus, there is no simple yes/no answer regarding whether to choose separate, combined or even integrated MR-PET. In the near future, different systems will be examined yielding versatile experience and probably new applications in both brain research and clinics.

\section{Conclusion \& future perspective}

The present results with first MR-PET prototypes demonstrate the feasibility of this new hybrid imaging technology and have led to an increasing interest of both researchers and clinicians. Although industrial MR-PET scanners produced in the near future will be constructed primarily for whole-body imaging, they will allow the development of new bimodal neuroimaging procedures. After further methodological developments, one may expect that reliable quantitative PET imaging will be possible with MR-PET. When reflecting whether MR-PET will have a similar success as PET/CT one must also consider that the soft tissue contrast of MRI is superior to CT and that MRI causes no radiation dose. Furthermore, MR-PET inherently possesses many degrees of freedom for future research and clinical applications by combining the specific capabilities of PET and MRI. However, the success of a new method is not independent of economic variables.

Financial \& competing interests disclosure
The authors have no relevant affiliations or financial
involvement with any organization or entity with a
financial interest in or financial conflict with the sub-
ject matter or materials discussed in the manuscript.
This includes employment, consultancies, honoraria,
stock ownership or options, expert testimony, grants or
patents received or pending, or royalties.
No writing assistance was utilized in the production
of this manuscript.

\section{Executive summary}

\section{Towards PET integrated with ultra-high MRI for small animal studies}

- For MR-PET in small animals different designs have been suggested based on either conventional PET technology or MR-insensitive solid-state electronics.

- The first applications demonstrate the potential of MR-PET in preclinical research.

First prototypes of a MR-PET scanner for human brain studies

- In the BrainPET's detectors photomultipliers are replaced by Avalanche photodiodes.

- The two imaging modalities of the 3TMR-BrainPET show no remarkable interferences.

First results obtained with $3 T M R-B r a i n P E T$

- The 3TMR-BrainPET allows PET simultaneously recorded with different MR sequences.

- The BrainPET offers an excellent image resolution.

- Bimodal imaging with an integrated MR-PET system increases patient's compliance.

The near future of MR-PET

- Experience with four prototypes is strongly considered in the ongoing development of whole-body MR-PET.

- The combination of MRI and PET in one scanner leads to new horizons of multiparametric functional neuroimaging.

\section{Bibliography}

Papers of special note have been highlighted as:

- of interest

-. of considerable interest

1. Beyer T, Townsend DW, Brun T: A combined PET/CT scanner for clinical oncology. J. Nucl. Med. 41, 1369-1379 (2000).
- Presents the primary suggestion of combining two different imaging technologies for complementary use in clinical diagnostics of tumor patients.

2. Bockisch A, Freudenberg LS, Schmidt D, Kuwert T: Hybrid imaging by SPECT/CT and PET/CT: proven outcomes in cancer imaging. Semin. Nucl. Med. 39, 276-289 (2009).
3. Delbeke D, Schöder H, Martin WH, Wahl RL: Hybrid imaging (SPECT/CT and PET/CT): improving therapeutic decisions. Semin. Nucl. Med. 39, 308-340 (2009).

4. Lonsdale MN, Beyer T: Dual-modality PET/CT instrumentation-today and tomorrow. Eur. J. Radiol. 73, 452-460 (2010). 
5. Hasegawa BH, Gingold EL, Reilly SM, Liew SC, Cann CE: Description of a simultaneous emission-transmission CT system. Proc. SPIE 1231, 50-60 (1990).

- Reports the primary description of a single photon emission computed tomography (SPECT)/CT.

6. Buck AK, Nekolla S, Ziegler $S$ et al.: SPECT/CT. J. Nucl. Med. 49, 1305-1319 (2008).

7. Shao Y, Cherry SR, Farahani K et al.: Simultaneous PET and MR imaging. Phys. Med. Biol. 42, 1965-1970 (1997).

- For the first time the feasibility of a PET study within MRI was demonstrated.

8. Raylman RR, Majewski S, Lemieux SK et al.: Simultaneous MRI and PET imaging of a rat brain. Phys. Med. Biol. 51, 371-379 (2006).

9. Lucas AJ, Hawkes RC, Ansorge et al: Development of a combined microPET-MR system. Technol. Cancer Res. Treat. 5, 337-341 (2006).

10. Mackewn JE, Strul D, Charles-Edwards G et al:: Performance evaluation and MR compatibility assessment of an optical fibre based pre-clinical MR-compatible PET scanner. IEEE NSS-MIC Record 4662-4666 (2008).

11. Yamamoto S, Mazumoto K, Senda M: Development of a multi-slice dual layer MR-compatible animal PET system using DOI detectors. J. Nucl. Med. 48(Suppl.), 89P (2007).

12. Handler WB, Gilbert KM, Peng H, Chronik BA: Simulation of scattering and attenuation of $511 \mathrm{keV}$ photons in a combined PET/field-cycled MRI system. Phys. Med. Biol. 51, 2479-2491 (2006).

13. Pichler B, Lorenz E, Mirzoyan R et al.: Performance test of a LSO-APD PET module in a 9.4 tesla magnet. IEEE NSS-MIC Record 1237-1239 (1998).

14. Pichler BJ, Judenhofer MS, Catana C et al.: Performance test of an LSO-APD detector in a 7-T MRI scanner for simultaneous PET/ MRI. J. Nucl. Med. 47, 639-647 (2006).

15. Catana C, Wu Y, Judenhofer MS, Qi J, Pichler BJ, Cherry SR: Simultaneous acquisition of multislice PET and MR images: initial results with a MR-compatible PET scanner. J. Nucl. Med. 47, 1968-1976 (2006).

- First Avalanche photodiodes (APD)-based MR-compatible PET detector for simultaneous PET and MRI in small animals is described. Different aspects of this hybrid approach and possible interferences between the two modalities are discussed comprehensively.
16. Judenhofer MS, Catana C, Swann BK et al:: PET/MR images, acquired with a compact MRI compatible PET detector in a 7 Tesla magnet. Radiology 244, 807-814 (2007).

- First description of a small animal MR-PET with direct coupling of scintillation crystals and APDs.

17. Judenhofer MS, Wehrl HF, Newport DF et al.: Simultaneous PET-MRI: a new approach for functional and morphological imaging. Nat. Med. 14, 459-465 (2008).

18. Wehrl HF, Judenhofer MS, Wiehr S, Pichler B: Pre-clinical PET/MR: technological advances and new perspectives in biomedical research. Eur. J. Nucl. Med. Mol. Imaging. 36 (Suppl. 1), S56-S68 (2009).

19. Schlyer D, Vaska P, Tomasi et al.: A simultaneous PET/MRI scanner based on RatCAP in small animals. IEEE NSS-MIC Record 3256-3259 (2007).

20. Maramraju SH, Smith SD, Stoll S et al.: Simultaneous PET/MR imaging results of a rat brain and a mouse heart with an MRI compatible PET detector in a $9.4 \mathrm{~T}$ microMRI. J. Nucl. Med. 51 (Suppl. 2), 47P (2010).

21. Schlemmer HP, Pichler B, Schmand M et al.: Simultaneous MR/PET imaging of the human brain: feasibility study. Radiology 248 , 1028-1035 (2008).

- Very first report demonstrating simultaneous MR-PET measurements in humans.

22. Schmand M, Burbar Z, Corbeil J et al.: Brain PET: first human tomograph for simultaneous (functional) PET and MR imaging. J. Nucl. Med. 48, 45P (2007).

23. Newport DF, Siegel SB, Swann BK et al.: QuickSilver: A flexible, extensible, and high-speed architecture for multimodality. IEEE NSS-MIC Record 2333-2334 (2006).

24. Herzog H, Tellmann L, Marx B et al: First performance tests of the 3TMR-BrainPET. J. Nucl. Med. 50 (Suppl. 2), 310P (2009).

25. de Jong HW, van Velden FH, Kloet RW et al:: Performance evaluation of the ECAT HRRT: an LSO-LYSO double layer high resolution, high sensitivity scanner. Phys. Med. Biol. 52, 1505-1526 (2007).

26. Piroth MD, Pinkawa M, Holy R et al.: Prognostic value of early ${ }^{18} \mathrm{~F}$-fluoroethyltyrosine PET after radiochemotherapy in glioblastoma multiforme. Int. J. Radiat. Oncol. Biol. Phys. DOI: 10.1016/j.ijrobp.2010.01.055 (2010) (Epub ahead of print).
27. Pauleit D, Floeth F, Hamacher $\mathrm{K}$ et al.: O- $\left(2-\left[{ }^{18} \mathrm{~F}\right]\right.$ fluoroethyl $)$-L-tyrosine PET combined with magnetic resonance imaging improves the diagnostic assessment of cerebral gliomas. Brain 128, 678-687 (2005).

28. Montandon ML, Zaidi H: Quantitative analysis of template-based attenuation compensation in 3D brain PET. Comput. Med. Imaging Graph. 31, 28-38 (2007).

29. Rota Kops E, Herzog H: Template based attenuation correction for PET in MR-PET scanners. IEEE NSS-MIC Record 3786-3789 (2008).

30. Hofmann M, Steinke F, Scheel V et al.: MRI-based attenuation correction for PET/ MRI: a novel approach combining pattern recognition and atlas registration. J. Nucl. Med. 49, 1875-1883 (2008).

31. Keereman V, Fierens Y, Broux T, De Deene Y, Lonneux M, Vandenberghe S: MRI-based attenuation correction for PET/MRI using ultrashort echo time sequences. J. Nucl. Med. 51, 812-818 (2010).

32. Catana C, van der Kouwe A, Benner T et al: Towards implementing an MR-based PET attenuation correction method for neurological studies on the MR-PET brain prototype. J. Nucl. Med. 51(9), 1431-1438 (2010).

33. Ashburner J, Friston KJ: Nonlinear spatial normalization using basic functions. Human Brain Mapping 7, 253-266 (1999).

34. Saraf-Lavi E, Bowen BC, Pattany PM, et al.: Proton MR spectroscopy of gliomatosis cerebri: case report of elevated myoinositol with normal choline levels. Am. J. Neuroradiol. 24, 946-951 (2003).

35. Boss A, Bisdas S, Kolb A, et al.: Hybrid PET/ MRI of intracranial masses: initial experiences and comparison to PET/CT. J. Nucl. Med. 51, 1198-1205 (2010).

-. Compares brain tumor imaging of 3TMR-BrainPET and PET/CT concluding that diagnostic imaging quality can be achieved using simultaneous hybrid PET/MR image acquisition.

36. Ratib O, Becker M, Vallee J et al: : Whole body PET-MRI scanner: first experience in oncology. J. Nucl. Med. 51(Suppl. 2), 46P (2010).

37. Lucignani G: PET-MRI synergy in molecular, functional and anatomical cancer imaging. Eur. J. Nucl. Med. Mol. Imaging 35, 1550-1553 (2008).

38. Antoch G, Bockisch A: Combined PET / MRI: a new dimension in whole-body oncology imaging? Eur. J. Nucl. Med. Mol. Imaging 36 (Suppl. S1), S113-S120 (2009). 
39. Heiss WD: The potential of PET/MR for brain imaging. Eur. J. Nucl. Med. Mol. Imaging 36(Suppl. S1), S105-S112 (2009).

40. Bolus NE, George R, Washington J, Newcomer BR: PET/MRI: the blendedmodality choice of the future? J. Nucl. Med. Technol. 37, 63-71 (2009).

41. Pichler B, Kolb A, Nagele T, Schlemmer HP: PET /MRI: paving the way for the next generation of clinical multimodality imaging applications. J. Nucl. Med. 51, 333-336 (2010).
42. Herzog H, Pietrzyk U, Shah NJ, Ziemons K: The current state, challenges and perspectives of MR-PET. NeuroImage 49, 2072-2082 (2010).

- Comprehensive overview on many aspects of the recent and current developments and status of MR-PET written as a critical review of an international workshop on MR-PET held in October 2008 at the Forschungszentrum Jülich, Germany.

\section{Website}

101. HYPER Image developing: A hybrid PET-MR (2008)

www.hybrid-pet-mr.eu 